\title{
Convergence of the Simplicial Rational Bernstein Form
}

\author{
Jihad Titi \\ University of Konstanz \\ Konstanz, Germany \\ jihadtiti@yahoo.com \\ Tareq Hamadneh \\ University of Konstanz \\ Konstanz, Germany \\ tareq.hamadneh@uni-konstanz.de \\ Jürgen Garloff \\ University of Applied Sciences / HTWG Konstanz \\ Konstanz, Germany \\ garloff@htwg-konstanz.de
}

\begin{abstract}
Bernstein polynomials on a simplex $V$ are considered. The expansion of a given polynomial $p$ into these polynomials provides bounds for the range of $p$ over $V$. Bounds for the range of a rational function over $V$ can easily obtained from the Bernstein expansions of the numerator and denominator polynomials of this function. In this paper it is shown that these bounds converge monotonically and linearly to the range of the rational function if the degree of the Bernstein expansion is elevated. If $V$ is subdivided then the convergence is quadratic with respect to the maximum of the diameters of the subsimplices.
\end{abstract}

Keywords: Bernstein polynomial-simplex·range bounds·rational function· degree elevation·subdivision

\section{Introduction}

During the last decade, polynomial minimization over simplices has attracted the interest of many researchers, see [1,2], [4], [6-12]. Special attention was paid to the use of the expansion of the given polynomial into Bernstein polynomials over a simplex, the so-called simplicial Bernstein expansion, [2], [4], [8], [10-12]. In [10-12], R. Leroy gave results on degree elevation and subdivision of the underlying simplex of this expansion. In [13] the Bernstein form of a polynomial over a box, the so-called tensorial Bernstein form, was used to find an enclosure of the range of a given (multivariate) 
rational function over a box. Convergence properties of this tensorial rational Bernstein form were investigated in [5]. In this paper we present convergence properties of the corresponding simplicial rational Bernstein form based on Leroy's results. The organization of our paper is as follows: In the next section we briefly recall the simplical polynomial Bernstein form and its basic properties, e.g., their range enclosing property. We also provide additional properties like sharpness, monotonicity, and convergence of the bounds which will be used later on. In Section 3 we present our main results, viz. convergence properties of the simplicial rational Bernstein form.

With the exception of the range enclosing property of the polynomial and rational forms, we state the results in each case only for the maximum of the quantities under consideration because the respective statements for the minimum are analogous.

\section{Bernstein Expansion over a Simplex}

In this section we present firstly the most important and fundamental properties of the Bernstein expansion over a simplex we will employ throughout the paper.

We follow the notation and definitions that have been used in [11], [12]. Firstly, we recall the definition of a simplex.

Definition 1. Let $\boldsymbol{v}_{0}, \ldots, \boldsymbol{v}_{n}$ be $n+1$ points of $\mathbb{R}^{n}$. The ordered list $V=\left[\boldsymbol{v}_{0}, \ldots, \boldsymbol{v}_{n}\right]$ is called simplex of vertices $\boldsymbol{v}_{0}, \ldots, \boldsymbol{v}_{n}$. The realization $|V|$ of the simplex $V$ is the set of $\mathbb{R}^{n}$ defined as the convex hull of the points $\boldsymbol{v}_{0}, \ldots, \boldsymbol{v}_{n}$. The diameter of $V$ is the length of the largest edge of $|V|$.

Throughout our paper we will assume that the points $\boldsymbol{v}_{0}, \ldots, \boldsymbol{v}_{n}$ are affinely independent in which case the simplex $V$ is non-degenerate. We will often consider the simplex $\Delta:=\left[\boldsymbol{0}, \boldsymbol{e}_{1}, \ldots, \boldsymbol{e}_{n}\right]$, called the standard simplex, where $\boldsymbol{O}$ is the zero vector in $\mathbb{R}^{n}$ and $\boldsymbol{e}_{i}$ is the $i^{t h}$ vector of the canonical basis of $\mathbb{R}^{n}, i=1, \ldots, n$. This is no restriction since any simplex $V$ in $\mathbb{R}^{n}$ can be mapped affinely upon $\Delta$.

Recall that any vector $x \in \mathbb{R}^{n}$ can be written as an affine combination of the vertices $\boldsymbol{v}_{0}, \ldots, \boldsymbol{v}_{n}$ with weights $\lambda_{0}, \ldots, \lambda_{n}$ called barycentric coordinates. If $\boldsymbol{x}=\left(x_{1}, \ldots, x_{n}\right)$ $\in \Delta$, then $\lambda=\left(\lambda_{0}, \ldots, \lambda_{n}\right)=\left(1-\sum_{i=1}^{n} x_{i}, x_{1}, \ldots, x_{n}\right)$.

For every multi-index $\alpha=\left(\alpha_{0}, \ldots, \alpha_{n}\right) \in \mathbb{N}^{n+1}$ and $\lambda=\left(\lambda_{0}, \ldots, \lambda_{n}\right) \in \mathbb{R}^{n+1}$ we write $|\alpha|:=\alpha_{0}+\ldots+\alpha_{n}$ and $\lambda^{\alpha}:=\prod_{i=0}^{n} \lambda_{i}^{\alpha_{i}}$. The relation $\leq$ on $\mathbb{N}^{n+1}$ is understood entrywise. For $\alpha, \beta \in \mathbb{N}^{n+1}$ with $\beta \leq \alpha$ we define

$$
\left(\begin{array}{l}
\alpha \\
\beta
\end{array}\right):=\prod_{i=0}^{n}\left(\begin{array}{l}
\alpha_{i} \\
\beta_{i}
\end{array}\right) .
$$

If $k$ is any natural number such that $|\alpha|=k$, we use the notation $\left(\begin{array}{l}k \\ \alpha\end{array}\right):=\frac{k !}{\alpha_{0} ! \cdots \alpha_{n} !}$.

Definition 2. [3, Section 8] Let $k$ be a natural number. The Bernstein polynomials of degree $k$ with respect to $V$ are the polynomials $\left(B_{\alpha}^{k}\right)_{|\alpha|=k}$, where

$$
B_{\alpha}^{k}:=\left(\begin{array}{l}
k \\
\alpha
\end{array}\right) \lambda^{\alpha} .
$$


The Bernstein polynomials of degree $k$ with respect to $V$ take nonnegative values on $V$ and sum up to $1: 1=\sum_{|\alpha|=k} B_{\alpha}^{k}$.

Let $p$ be a polynomial of degree $l$,

$$
p(x)=\sum_{|\beta| \leq l} a_{\beta} x^{\beta} .
$$

Since the Bernstein polynomials of degree $k$ form a basis of the vector space $\mathbb{R}_{k}[\boldsymbol{X}]$ of polynomials of degree at most $k$, see, e.g, [10, Proposition 1.6], $p$ can be uniquely expressed as $(l \leq k)$

$$
p(\boldsymbol{x})=\sum_{|\alpha|=k} b_{\alpha}(p, k, V) B_{\alpha}^{k}
$$

The numbers $b_{\alpha}(p, k, V)$ are called the Bernstein coefficients of $p$ of degree $k$ with respect to $V$. If $V=\Delta$, we obtain by multinomial expansion the following representation of the Bernstein coefficients in terms of the coefficients of $p(|\alpha|=k)$

$$
b_{\alpha}(p, k, \Delta)=\sum_{\beta \leq \alpha} \frac{\left(\begin{array}{l}
\alpha \\
\beta
\end{array}\right)}{\left(\begin{array}{c}
k \\
\beta
\end{array}\right)} a_{\beta} .
$$

It is easy to see from (3) that the Bernstein coefficients are linear with respect to $p$.

Definition 3. Let $V=\left[\boldsymbol{v}_{0}, \ldots, \boldsymbol{v}_{n}\right]$ be a non-degenerate simplex of $\mathbb{R}^{n}$ and $p \in \mathbb{R}_{l}[\boldsymbol{X}]$.

- The grid points of degree $k$ associated to $V$ are the points $(|\alpha|=k)$

$$
\boldsymbol{v}_{\alpha}(k, V):=\frac{1}{k}\left(\alpha_{0} \boldsymbol{v}_{0}+\ldots+\alpha_{n} \boldsymbol{v}_{n}\right) \in \mathbb{R}^{n}
$$

- The control points associated to $p$ of degree $k$ with respect to $V$ are the points $\left(\boldsymbol{v}_{\alpha}(k, V), b_{\alpha}(p, k, V)\right) \in \mathbb{R}^{n+1}$.

The set of control points of p forms its control net of degree $k$.

- The discrete graph of $p$ of degree $k$ with respect to $V$ is formed by the collection $\left(\boldsymbol{v}_{\alpha}(k, V), p\left(\boldsymbol{v}_{\alpha}(k, V)\right)\right)_{|\alpha|=k}$.

In the sequel, $\left(\boldsymbol{e}_{0}, \ldots, \boldsymbol{e}_{n}\right)$ denotes the standard basis of $\mathbb{R}^{n+1}$.

Proposition 1. [11, Proposition 2.7] For $p \in \mathbb{R}_{l}[\boldsymbol{X}]$ the following properties hold.

(i) Interpolation at the vertices:

$$
b_{k \mathbf{e}_{i}}=p\left(\boldsymbol{v}_{i}\right), \quad 0 \leq i \leq n ;
$$

(ii) convex hull property: the graph of $p$ over $V$ is contained in the convex hull of its associated control points;

(iii) range enclosing property:

$$
\min _{|\alpha|=k} b_{\alpha}(p, k, V) \leq p(\boldsymbol{x}) \leq \max _{|\alpha|=k} b_{\alpha}(p, k, V), \boldsymbol{x} \in V .
$$


The following proposition gives necessary and sufficient conditions when equality holds in (6).

Proposition 2. Let $p \in \mathbb{R}_{l}[\boldsymbol{X}]$. Then

$$
\max _{\boldsymbol{x} \in \Delta} p(\boldsymbol{x})=\max _{|\alpha|=k} b_{\alpha}(p, k, \Delta)
$$

if and only if

$$
\max _{|\alpha|=k} b_{\alpha}(p, k, \Delta)=b_{\alpha^{*}}(p, k, \Delta) \text { for some } \alpha^{*}=k \boldsymbol{e}_{i_{0}}, i_{0} \in\{0, \ldots, n\} .
$$

A similar statement holds for the minimum.

Proof. Without loss of generality we consider the standard simplex (of dimension $n$ ). If the maximum is attained at an index $k \boldsymbol{e}_{i_{0}}$ then the statement holds trivially by Proposition 1 (i). Conversely, suppose first that the $b_{\alpha}(p, k, \Delta)$ are equal for all $|\alpha|=k$. Then the statement is trivial. Otherwise not all $b_{\alpha}(p, k, \Delta)$ are equal. Suppose that the maximum of $p(\boldsymbol{x})$ occurs at $\boldsymbol{x}^{*} \in \Delta$ with barycentric coordinates $\boldsymbol{\lambda}^{*}$. If $\boldsymbol{x}^{*}$ is in the interior of $\Delta$ then $0<B_{\alpha}^{k}\left(\lambda^{*}\right)<1$ for all $|\alpha|=k$ and hence

$$
\begin{aligned}
p\left(\boldsymbol{x}^{*}\right) & =\sum_{|\alpha|=k} b_{\alpha}(p, k, \Delta) B_{\alpha}^{k}\left(\lambda^{*}\right) \\
& <\max _{|\alpha|=k} b_{\alpha}(p, k, \Delta) \sum_{|\alpha|=k} B_{\alpha}^{k}\left(\lambda^{*}\right)=\max _{|\alpha|=k} b_{\alpha}(p, k, \Delta),
\end{aligned}
$$

a contraction. If $\boldsymbol{x}^{*}$ is lying on the boundary of $\Delta$ then it is contained in a subsimplex of dimension $n-1, \Delta^{\prime}$ say. The Bernstein coefficients of $p$ over $\Delta^{\prime}$ coincide with the respective coefficients contained in the part of the array $\left(b_{\alpha}(p, k, \Delta),|\alpha|=k\right)$ between the (extreme) coefficients associated with the vertices of $\Delta^{\prime}$ according to (5). Now we can apply the arguments used above to $\Delta^{\prime}$. Continuing in this way of examining all possible cases, we decrease the dimension of the simplices to be investigated step by step and arrive finally at the situation in which $x^{*}$ is a vertex of $\Delta$ which completes the proof.

Recall that the barycentric coordinates can be written in terms of the components of the variable $x \in \mathbb{R}^{n}$. By multiplying both sides of (2) with $1=\sum_{i=0}^{n} \lambda_{i}=$ $\left(1-\sum_{i=1}^{n} x_{i}\right)+\sum_{i=1}^{n} x_{i}$ and rearranging the result we obtain, see also [10, Proposition $1.12]$,

$$
p(\boldsymbol{x})=\sum_{|\beta|=k+1} b_{\beta}(p, k+1, V) B_{\beta}^{k+1},
$$

where

$$
\begin{aligned}
b_{\beta}(p, k+1, V)=b_{\alpha+\boldsymbol{e}_{l}}(p, k+1, V) & =\frac{1}{k+1} \sum_{i=0, i \neq l}^{n} \alpha_{i} b_{\alpha+\boldsymbol{e}_{l}-\boldsymbol{e}_{i}}(p, k, V) \\
& +\frac{\alpha_{l}+1}{k+1} b_{\alpha}(p, k, V) .
\end{aligned}
$$


It is easy to see from (10) that the coefficients $b_{\beta}(p, k+1, V)$ are convex combinations of the coefficients $b_{\alpha}(p, k, V)$. Hence the upper bounds decrease monotonically.

Proposition 3. Let $p \in \mathbb{R}_{l}[\boldsymbol{X}]$. Then it holds that

$$
\max _{|\beta|=k+1} b_{\beta}(p, k+1, V) \leq \max _{|\alpha|=k} b_{\alpha}(p, k, V)
$$

In order to relate the control net and the discrete graph of a given polynomial, $\mathrm{R}$. Leroy [11], [12] suggested to use the so-called second differences which are given in the following definition.

Definition 4. Let $V=\left[\boldsymbol{v}_{0}, \ldots, \boldsymbol{v}_{n}\right]$ be a non-degenerate simplex of $\mathbb{R}^{n}$. For $|\gamma|=k-2$ and $0 \leq i<j \leq n$, define the second differences of $p$ of degree $k$ with respect to $V$ as

$$
\nabla^{2} b_{\gamma, i, j}(p, k, V):=b_{\gamma+\boldsymbol{e}_{i}+\boldsymbol{e}_{j-1}}+b_{\gamma+\boldsymbol{e}_{i-1}+\boldsymbol{e}_{j}}-b_{\gamma+\boldsymbol{e}_{i-1}+\boldsymbol{e}_{j-1}}-b_{\gamma+\boldsymbol{e}_{i}+\boldsymbol{e}_{j}},
$$

with the convention $\boldsymbol{e}_{-1}:=\boldsymbol{e}_{n}$. The second differences constitute the collection

$$
\nabla^{2} b(p, k, V):=\left(\nabla^{2} b_{\gamma, i, j}(p, k, V)\right)_{|\gamma|=k-2,0 \leq i<j \leq n} .
$$

The maximum of the second differences, i.e.,

$$
\left\|\nabla^{2} b(p, k, V)\right\|_{\infty}=\max _{|\gamma|=k-2,0 \leq i<j \leq n}\left|\nabla^{2} b_{\gamma, i, j}(p, k, V)\right|
$$

will play an important role in the subsequent convergence analysis.

The following theorem gives the convergence of the control net to the discrete graph of a given polynomial with respect to degree elevation. Since any simplex can be mapped upon the standard simplex by an affine transformation, we present the following statements only for $\Delta$.

Theorem 1. [12, Theorem 4.2] Let $p \in \mathbb{R}_{l}[\boldsymbol{X}]$ and $l<k$. Then

$$
\max _{|\alpha|=k}\left|p\left(\boldsymbol{v}_{\alpha}(k, \Delta)\right)-b_{\alpha}(p, k, \Delta)\right| \leq \frac{n(n+2) l(l-1)}{24(k-1)}\left\|\nabla^{2} b(p, l, \Delta)\right\|_{\infty} .
$$

The following corollary is an immediate consequence of Theorem 1.

Corollary 1. Let $p \in \mathbb{R}_{l}[\boldsymbol{X}]$. If $l<k$, then

$$
\max _{|\alpha|=k} b_{\alpha}(p, k, \Delta)-\max _{\boldsymbol{x} \in \Delta} p(\boldsymbol{x}) \leq \frac{T_{1}}{k-1}
$$

where

$$
T_{1}:=\frac{n(n+2) l(l-1)}{24}\left\|\nabla^{2} b(p, l, \Delta)\right\|_{\infty}
$$


Proof. Assume that the maximum of $\left(b_{\alpha}(p, k . \Delta)\right)$ such that $|\alpha|=k$ is attained at $b_{\alpha^{*}}(p, k, \Delta)$. Then we have

$$
\begin{aligned}
\max _{|\alpha|=k} b_{\alpha}(p, k, \Delta)-\max _{\boldsymbol{x} \in \Delta} p(\boldsymbol{x}) & \leq b_{\alpha^{*}}(p, k, \Delta)-p\left(\boldsymbol{v}_{\alpha^{*}}\right) \\
& =\left|b_{\alpha^{*}}(p, k, \Delta)-p\left(\boldsymbol{v}_{\alpha^{*}}\right)\right| \leq \frac{T_{1}}{k-1} .
\end{aligned}
$$

The second inequality follows since $\boldsymbol{v}_{\alpha^{*}}$ is a grid point in $\Delta$, while the last inequality follows by using Theorem 1 .

Definition 5. Let $V=\left[\boldsymbol{v}_{0}, \ldots, \boldsymbol{v}_{n}\right]$ be a non-degenerate simplex of $\mathbb{R}^{n}$ and $\boldsymbol{v}^{\prime} \in \mathbb{R}^{n}$. The simplices $V^{i}$ generated by subdivision with respect to the point $\boldsymbol{v}^{\prime}$ are defined as

$$
V^{i}:=\left[\boldsymbol{v}_{0}, \ldots, \boldsymbol{v}_{i-1}, \boldsymbol{v}^{\prime}, \boldsymbol{v}_{i+1}, \ldots, \boldsymbol{v}_{n}\right], 0 \leq i \leq n .
$$

Assume that $\Delta$ has been subdivided with respect to a point $\boldsymbol{v}^{\prime}$ in $\Delta, \Delta=V^{1} \cup \ldots \cup$ $V^{\sigma}$ say. By using [12, Algorithm 4.6 (de Casteljau)] it is easy to see that the Bernstein coefficients of $p$ over any $V^{i}$ are contained in the interval $\left[\min _{|\alpha|=k} b_{\alpha}(p, k, \Delta)\right.$, $\left.\max _{|\alpha|=k} b_{\alpha}(p, k, \Delta)\right], 1 \leq i \leq \sigma$, see [12, Remark 4.7]. The following theorem gives the convergence of the control net to the discrete graph with respect to subdivision.

Theorem 2. [12, Theorem 4.9] Let $\Delta=V^{1} \cup \ldots \cup V^{\sigma}$ be a subdivision of the standard simplex $\Delta$ and $h$ be an upper bound on the diameters of the $V^{i}$ 's. Then, for each $i \in$ $\{1, \ldots, \sigma\}$ and $|\alpha|=k$, we have

$$
\left|p\left(\boldsymbol{v}_{\alpha}\left(k, V^{i}\right)\right)-b_{\alpha}\left(p, k, V^{i}\right)\right| \leq h^{2} k \frac{n^{2}(n+1)(n+2)^{2}(n+3)}{576}\left\|\nabla^{2} b(p, k, \Delta)\right\|_{\infty} .
$$

The following corollary can similarly be proven as Corollary 1.

Corollary 2. Let $p \in \mathbb{R}_{l}[\boldsymbol{X}], \Delta=V^{1} \cup \ldots \cup V^{\sigma}$ be a subdivision of the standard simplex $\Delta$ and $h$ be an upper bound on the diameters of the $V^{i}$ 's. Then

$$
\max _{\substack{|\alpha|=k, i=1, \ldots, \sigma}} b_{\alpha}\left(p, k, V^{i}\right)-\max _{\boldsymbol{x} \in \Delta} p(\boldsymbol{x}) \leq h^{2} T_{2},
$$

where

$$
T_{2}:=k \frac{n^{2}(n+1)(n+2)^{2}(n+3)}{576}\left\|\nabla^{2} b(p, k, \Delta)\right\|_{\infty}
$$

\section{The Simplicial Rational Bernstein Form}

In this section we present our results in the case of rational functions. Throughout this section we assume that $p$ and $q$ are polynomials of degree less than or equal to $l$ with Bernstein coefficients $b_{\alpha}(p, k, \Delta)$ and $b_{\alpha}(q, k, \Delta),|\alpha|=k$, respectively, over the standard simplex $\Delta$, where $l \leq k$. We also assume that all Bernstein coefficients $b_{\alpha}(q, k, \Delta)$ have the same sign and are non-zero (this implies that $q(x) \neq 0$, for all $\boldsymbol{x} \in \Delta$ ) and without loss of generality we may assume that all of them are positive. 
For the negative case replace $q$ by $-q$. The following theorem provides bounds for the range of $f:=\frac{p}{q}$ over $\Delta$. We use the notation

$$
b_{\alpha}(f, k, V):=\frac{b_{\alpha}(p, k, V)}{b_{\alpha}(q, k, V)} \text { for all } \alpha,|\alpha|=k .
$$

Theorem 3. [13, Theorem 3.1, Remark 6] The range off over $\Delta$ can be bounded by

$$
\min _{|\alpha|=k} b_{\alpha}(f, k, \Delta) \leq f(\boldsymbol{x}) \leq \max _{|\alpha|=k} b_{\alpha}(f, k, \Delta), \boldsymbol{x} \in \Delta .
$$

We now extend results from the polynomial to the rational case.

Theorem 4. The equality holds in the right inequality in (19) if and only if

$$
\max _{|\alpha|=k} b_{\alpha}(f, k, \Delta)=b_{\alpha^{*}}(f, k, \Delta) \text { for some } \alpha^{*}=k \boldsymbol{e}_{i_{0}} i_{0} \in\{0, \ldots, n\} .
$$

A similar statement holds for the equality in the left inequality.

Proof. Put $M:=\max _{\boldsymbol{x} \in \Delta} f(\boldsymbol{x})$. Suppose that the equality holds in the right inequality of (19). Then there exists $x^{*} \in \Delta$ and $\alpha^{*}$ with $\left|\alpha^{*}\right|=k$ such that $f\left(x^{*}\right)=M=$ $\max _{|\alpha|=k} b_{\alpha}(f, k, \Delta)=b_{\alpha^{*}}(f, k, \Delta)$. Hence $s(\boldsymbol{x}):=p(\boldsymbol{x})-M q(\boldsymbol{x}) \leq 0$ for all $\boldsymbol{x} \in \Delta$, $b_{\alpha}(s, k, \Delta) \leq 0$ for all $\alpha$ with $|\alpha|=k$ (by linearity of the Bernstein coefficients), and $s\left(\boldsymbol{x}^{*}\right)=0$. By Propositions 1 (iii) and 2 there exists $i_{0} \in\{0, \ldots, n\}$ such that $\alpha^{*}=k \boldsymbol{e}_{i_{0}}$ and $s\left(x^{*}\right)=b_{\alpha^{*}}(s, k, \Delta)=0$. By linearity, $b_{\alpha^{*}}(p, k, \Delta)=M b_{\alpha^{*}}(q, k, \Delta)$. Hence the first implication follows. The converse holds by Proposition 1 (i).

Theorem 5. The upper bounds decrease monotonically

$$
\max _{|\beta|=k+1} b_{\beta}(f, k+1, \Delta) \leq \max _{|\alpha|=k} b_{\alpha}(f, k, \Delta)
$$

Proof. Put

$$
M^{(k)}:=\max _{|\alpha|=k} b_{\alpha}(f, k, \Delta), \text { and } s(\boldsymbol{x}):=p(\boldsymbol{x})-M^{(k)} q(\boldsymbol{x}) .
$$

Then by the linearity of the Bernstein coefficients, we have for all $\beta$ with $|\beta|=k+1$

$$
\begin{aligned}
b_{\beta}(s, k+1, \Delta) & \leq \max _{|\beta|=k+1}\left(b_{\beta}(p, k+1, \Delta)-M^{(k)} b_{\beta}(q, k+1, \Delta)\right) \\
& \leq \max _{|\alpha|=k}\left(b_{\alpha}(p, k, \Delta)-M^{(k)} b_{\alpha}(q, k, \Delta)\right) \leq 0 .
\end{aligned}
$$

The second inequality follows by application of Proposition 3 to the polynomial $s$ and the last inequality is a consequence of the definition of $M^{(k)}$. This implies

$$
b_{\beta}(p, k+1, \Delta) \leq M^{(k)} b_{\beta}(q, k+1, \Delta)
$$

from which the result follows.

Now we turn to the convergence of the bounds for the range of rational functions provided by the Bernstein coefficients under degree elevation and subdivision. 
Theorem 6. For $l<k$ it holds that

$$
\max _{|\alpha|=k} b_{\alpha}(f, k, \Delta)-\max _{x \in \Delta} f(x) \leq \frac{A_{1}}{k-1},
$$

where

$$
A_{1}:=\frac{n(n+2) l(l-1)}{24 B_{1}}\left(\left\|\nabla^{2} b(p, l, \Delta)\right\|_{\infty}+\max _{|\alpha|=l}\left|b_{\alpha}(f, l, \Delta)\right|\left\|\nabla^{2} b(q, l, \Delta)\right\|_{\infty}\right)
$$

and $B_{1}:=\min _{|\alpha|=l} b_{\alpha}(q, l, \Delta)$.

Proof. The proof follows by using Corollary 1 and arguments similar to that given in the proof of the following theorem.

Remark 1. For any $0<\epsilon$ we can guarantee that

$$
\max _{|\alpha|=k} b_{\alpha}(f, k, \Delta)-\max _{\boldsymbol{x} \in \Delta} f(\boldsymbol{x})<\epsilon
$$

if we choose $\frac{A_{1}}{\epsilon}+1<k$.

The last theorem shows that the convergence of the bounds to the range is only linear (in $k$ ) if we elevate the degree. Instead, if we subdivide $\Delta$ we obtain quadratic convergence with respect to the maximum diameter of the subsimplices.

Theorem 7. Let $\Delta=V^{1} \cup \ldots \cup V^{\sigma}$ be a subdivision of the standard simplex $\Delta$ and $h$ be an upper bound on the diameters of the $V^{i}$ 's. Then we have

$$
\max _{\substack{|\alpha|=k, i=1, \sigma}} b_{\alpha}\left(f, k, V^{i}\right)-\max _{\boldsymbol{x} \in \Delta} f(\boldsymbol{x}) \leq h^{2} A_{2}
$$

where

$$
A_{2}:=k \frac{n^{2}(n+1)(n+2)^{2}(n+3)}{576 B_{1}}\left(\left\|\nabla^{2} b(p, k, \Delta)\right\|_{\infty}+B_{2}\left\|\nabla^{2} b(q, k, \Delta)\right\|_{\infty}\right),
$$

$B_{1}$ is given in Theorem 6 , and $B_{2}:=\frac{\max _{|\alpha|=k}\left|b_{\alpha}(p, k, \Delta)\right|}{B_{1}}$.

Proof. Suppose that $\max _{|\alpha|=k,} b_{\alpha}\left(f, k, V^{i}\right)$ is attained at $b_{\alpha^{*}}\left(f, k, V^{i_{0}}\right)$ with $\left|\alpha^{*}\right|=k$, $i_{0} \in\{1, \ldots, \sigma\}$. Then

$$
\begin{aligned}
& \max _{\substack{|\alpha|=k, i=1, \ldots, \sigma}} b_{\alpha}\left(f, k, V^{i}\right)-\max _{\boldsymbol{x} \in \Delta} f(\boldsymbol{x}) \leq b_{\alpha^{*}}\left(f, k, V^{i_{0}}\right)-\max _{\boldsymbol{x} \in V^{i_{0}}} f(\boldsymbol{x}) \\
\leq & \left|\frac{\left[p\left(\boldsymbol{v}_{\alpha^{*}}\left(k, V^{i_{0}}\right)\right)-b_{\alpha^{*}}\left(p, k, V^{i_{0}}\right)\right]-b_{\alpha^{*}}\left(f, k, V^{i_{0}}\right)\left[q\left(\boldsymbol{v}_{\alpha^{*}}\left(k, V^{i_{0}}\right)\right)-b_{\alpha^{*}}\left(q, k, V^{i_{0}}\right)\right]}{q\left(\boldsymbol{v}_{\alpha^{*}}\left(k, V^{i_{0}}\right)\right)}\right| \\
\leq & \frac{\left|p\left(\boldsymbol{v}_{\alpha^{*}}\left(k, V^{i_{0}}\right)\right)-b_{\alpha^{*}}\left(p, k, V^{i_{0}}\right)\right|+\left|b_{\alpha^{*}}\left(f, k, V^{i_{0}}\right)\right|\left|q\left(\boldsymbol{v}_{\alpha^{*}}\left(k, V^{i_{0}}\right)\right)-b_{\alpha^{*}}\left(q, k, V^{i_{0}}\right)\right|}{\left|q\left(\boldsymbol{v}_{\alpha^{*}}\left(k, V^{i_{0}}\right)\right)\right|} \\
\leq & h^{2} A_{2},
\end{aligned}
$$


where the second inequality follows since $\boldsymbol{v}_{\alpha^{*}}\left(k, V^{i_{0}}\right)$ is a grid point in $V^{i_{0}}$, the third follows by using the triangle inequality, and the fourth is a consequence of Theorem 2 and the fact that the Bernstein coefficients of a polynomial over $V^{i_{0}}$ are contained in the interval spanned by the Bernstein coefficients over $\Delta$.

We conclude the paper with a lower bound on the number of subdivision steps needed in order to obtain a tolerance $\epsilon>0$ between the maximum of the Bernstein coefficients of the given rational function over the subsimplices and its maximum over $\Delta$. Before we present our result we need the following definition.

Definition 6. [12, Definition 5.5] Let $V$ be a non-degenerate simplex of $\mathbb{R}^{n}, S(V)$ be a subdivision of the simplex $V$, i.e., $S(V)=\left(V^{1}, \ldots, V^{\sigma}\right)$ with $V=V^{1} \cup \ldots \cup V^{\sigma}$.

- By $m(S(V))$ the largest diameter of the subsimplices $V^{i}$ is denoted.

- The subdivision scheme $S$ is said to have a shrinking factor $C, 0 \leq C \leq 1$, if for every simplex $V$,

$$
m(S(V)) \leq C m(V)
$$

Theorem 8. Let $S$ be a subdivision scheme with shrinking factor $C, 0<C<1$. Then

$$
\max _{\substack{|\alpha|=k, i=1, \ldots, \sigma}} b_{\alpha}\left(f, k, V^{i}\right)-\max _{\boldsymbol{x} \in \Delta} f(\boldsymbol{x})<\epsilon,
$$

if $\frac{\ln \frac{\epsilon}{2 A_{2}}}{2 \ln C}<N$, where $A_{2}$ is given in Theorem 7 .

Proof. For any $0<\epsilon$ take $N$ such that $2 C^{2 N} A_{2}<\epsilon$. By using Definition 6 and the fact that $m(\Delta)=\sqrt{2}$ we may choose $h=\sqrt{2} C^{N}$. Hence by using Theorem 7 and $0<C<1$ the result follows.

\section{References}

1. Basu, S., Leroy, R., Roy, M.-F:: A Bound on the Minimum of a Real Positive Polynomial over the Standard Simplex, arxiv: 0902.3304v1, Feb 19, 2009, available at www.arxiv.org

2. Boudaoud, F., Caruso, F., Roy, M.-F.: Certificates of Positivity in the Bernstein Basis, Discrete Comput. Geom. 39, 639-655 (2008)

3. Farouki, R.T.: The Bernstein Polynomial Basis: A Centennial Retrospective. Comput. Aided Geom. Design 29, 379-419 (2012)

4. Garloff, J.: Convergent Bounds for the Range of Multivariate Polynomials.In: Nickel, K.(ed.) Interval Mathematics 1985. LNCS, vol. 212, pp. 37-56. Springer, Heidelberg (1986)

5. Garloff, J., Hamadneh, T.: Convergence and Inclusion Isotonicity of the Tensorial Rational Bernstein Form. In: Tucker, W., Wolff von Guddenberg, J. (eds), Proceedings of the 16th GAMM-IMACS International Symposium on Scientific Computing, Computer Arithmetic and Validated Numerics, LNCS, Springer, Heidelberg, to appear

6. Jeronimo, G., Perrucci, D.: On the Minimum of a Positive Polynomial over the Standard Simplex, J. Symbolic Comput. 45, 434-442 (2010)

7. de Klerk, E.: The complexity of Optimizing over a Simplex, Hypercube or Sphere: a Short Survey, CEJOR Cent. Eur. J. Oper. Res. 16, 111-125 (2008) 
8. de Klerk, E., den Hertog, D., Elabwabi, G.: On the Complexity of Optimization over the Standard Simplex, European J. Oper. Res. 191, 773-785 (2008)

9. de Klerk, E., Laurent, M., Parrilo, P.A., A PTAS for the Minimization of Polynomials of Fixed Degree over the Simplex, Theor. Comput. Sci. 361, 210-225 (2006)

10. Leroy, R.: Certificats de positivité et minimisation polynomiale dans la base de Bernstein multivariée, Ph D Thesis, Université de Rennes 1 (2008)

11. Leroy, R.: Convergence under Subdivision and Complexity of Polynomial Minimization in the Simplicial Bernstein Basis, Reliab. Comput. 17, 11-21 (2012)

12. Leroy, R.: Certificates of Positivity in the Simplicial Bernstein Basis, preprint. http:hal.archives-ouvertes.fr/hal-00589945 $v_{1} /$, May 3, 2011

13. Narkawicz, A., Garloff, J., Smith, A.P., Muñoz, C.A.: Bounding the Range of a Rational Function over a Box. Reliab. Comput. 17, 34-39 (2012) 